\title{
ZERO RESIDUAL POLARIZATION OF NEGATIVE MUONS IMPLANTED IN $\mathrm{C}_{60}$
}

\section{A. SCHENCK, F.N. GYGAX, A. AMATO and R. FEYERHERM} Institute for Intermediate Energy Physics, Swiss Federal Institute of Technology (ETH) Zürich, CH-5232 Villigen PSI, Switzerland

T.J.S. DENNIS, R.M. MACRAE and K. PRASSIDES

School of Chemistry and Molecular Sciences, University of Sussex, Brighton $B N I$ gQJ, U.K.

We report preliminary results on $\mu^{-}$-depolarization in $\mathrm{C}_{60}$. In contrast to graphite and diamond no clear $\mu^{-\mathrm{SR}}$ signal could be detected in TF-measurements in $\mathrm{C}_{60}$.

Since the discovery of parity violation in the $\pi-\mu-e$ decay chain it is well known that negative muons $\left(\mu^{-}\right)$implanted in graphite will preserve about $1 / 6$ of the initial polarization when cascading down to the lowest Bohr orbital $\left(1{ }^{2} \mathrm{~S}_{1 / 2}\right)$ after capture by a $\mathrm{C}$-atom [1]. The loss of polarization during the cascade is well understood on the basis of spin orbit coupling [2]. Since the ${ }_{6}^{12} \mathrm{C}$-nucleus has zero spin no further depolarization takes place in the ground state provided the electron shell is also in a zero total angular momentum state. Recent measurements by the Stuttgart group at PSI have also shown that $\mu^{-}$implanted in diamond display a residual polarization of about $1 / 6$ of its initial value [3].

Implanting polarized $\mu^{-}$into the fullerene $\mathrm{C}_{60}$ (containing $10 \% \mathrm{C}_{70}$ ) we made the very surprising observation that practically no residual polarization was left in the ground state. Fig. 1 shows a Fourjer spectrum obtained from data taken in pyrolytic graphite and Fig. 2 a spectrum in $\mathrm{C}_{60}$, both for a transverse field of $100 \mathrm{mT}$ and $300 \mathrm{~K}$. As can be seen there is a sizable $\mu^{-}$-precession signal in graphite (fitted asymmetry $4.3(2) \%$, no relaxation) and essentially no signal in $\mathrm{C}_{60}$ (fitted asymmetry $0.9(4) \%$, relaxation rate $\lambda \approx 0.5 \mu \mathrm{s}^{-1}$ - the spectrometer would yield an asymmetry of $\approx 24 \%$ for muons conserving the full polarization). Essentially the same result was obtained at $12 \mathrm{~K}$ (Fig. 3). 


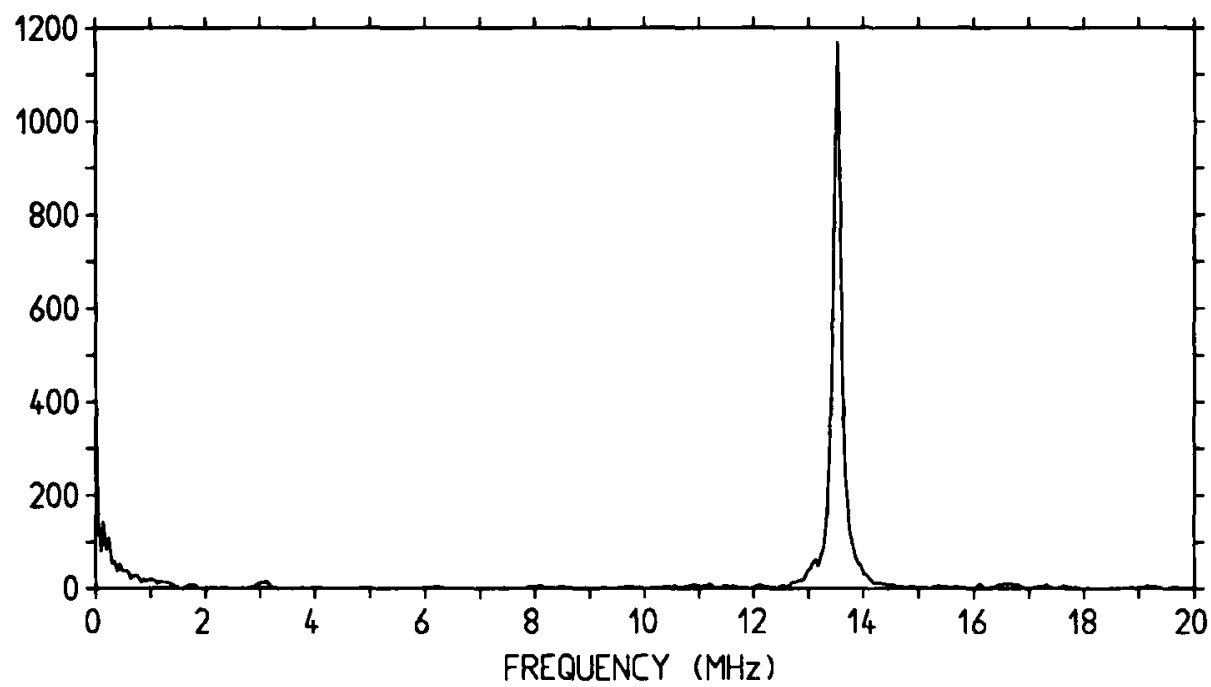

Fig. 1. Calibration in graphite at $100 \mathrm{mT}$. Fourier power obtained from a $\mu \mathrm{SR}$ histogram with negative muons in a pyrolytic graphite sample for a transverse field of $100 \mathrm{mT}$. The fitted signal at $13.5 \mathrm{MHz}$ shows an amplitude $A=4.3(2) \%$ and no relaxation.

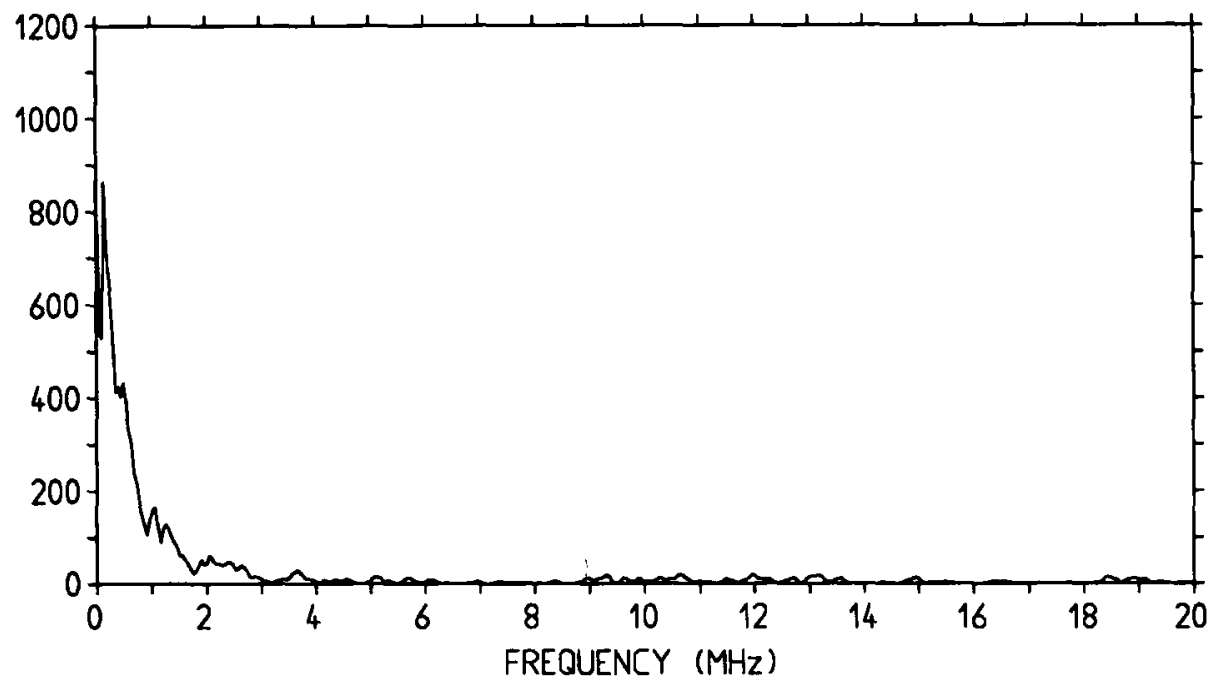

Fig. 2. Fourier power for $\mu^{-}$in a $\mathrm{C}_{60}$ sample (with $10 \% \mathrm{C}_{70}$ ) at $100 \mathrm{mT}$ and $300 \mathrm{~K}$. The $13.5 \mathrm{MHz}$ signal is much weaker than in graphite. A fit gives $A=0.9(4) \%$ with a relaxation $\lambda$ of approximately $0.5 \mu \mathrm{s}^{-1}$. 
This result seems to imply that the $\mu^{-}$in the 1 s ground state is exposed to a strong hyperfine interaction, since it is unlikely that the cascade is different from the one in graphite. Two possibilities come to mind: (i) the formed pseudo-atom with valence 3 remains in the $\mathrm{C}_{60}$ shell, but a double bond to one of the C-neighbours is broken and a chemical radical is formed with the unpaired electron coupling to the $\mu^{-}$; (ii) the formed pseudo-B atom is displaced to the center of the remaining cage where the neutral paramagnetic " $B$ " with an unpaired electron in the 2 -state may be rather stable. The remaining $\mathrm{C}_{59}$ shell may not be stable but could rearrange to form a $\mathrm{C}_{58}$ shell with one $\mathrm{C}$-atom expelled. Which possibility is realized is a question of energies. While the binding energy of a C-atom to $\mathrm{C}_{60}$ is of the order of $0.6 \mathrm{eV} \mathrm{[4]} \mathrm{the} \mathrm{recoil} \mathrm{energy} \mathrm{transmitted} \mathrm{to} \mathrm{muonic} \mathrm{carbon} \mathrm{in} \mathrm{the}$ $2 \mathrm{p}-1 \mathrm{~s}$ muonic transition $(75 \mathrm{keV}$ ) is $0.25 \mathrm{eV}$. The two energies are so close that neither of the two possibilities can be easily ruled out. In both cases, $\mu \mathrm{SR}$ signals similar to those observed upon $\mu^{+}$implantation are expected.

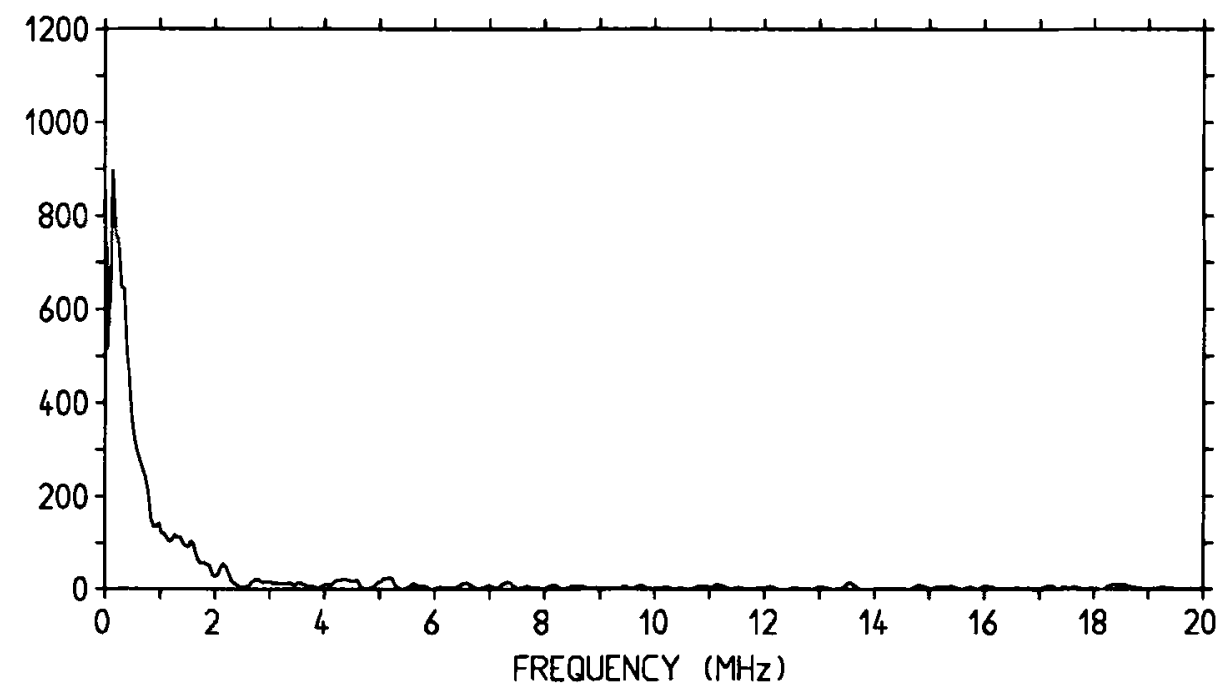

Fig. 3. Fourier power for $\mu^{-}$in the $\mathrm{C}_{60}$ sample at $100 \mathrm{mT}$ and $12 \mathrm{~K}$. The $13.5 \mathrm{MHz}$ signal, with $A=1.0(4) \%$ and $\lambda \approx 0.5 \mu \mathrm{s}^{-1}$, is insignificantly stronger than at $300 \mathrm{~K}$ (Fig. 2).

However, low transverse field $(0.8 \mathrm{mT})$ measurements at room temperature did not reveal any sign of a muonium or radical-like precession pattern. This can be seen from Fig. 4, which shows another Fourier transform of the $\mu^{-}$SR signal in $\mathrm{C}_{60}$, but this time at $0.8 \mathrm{mT}$. No precession frequency is indicated up to $250 \mathrm{MHz}$. 


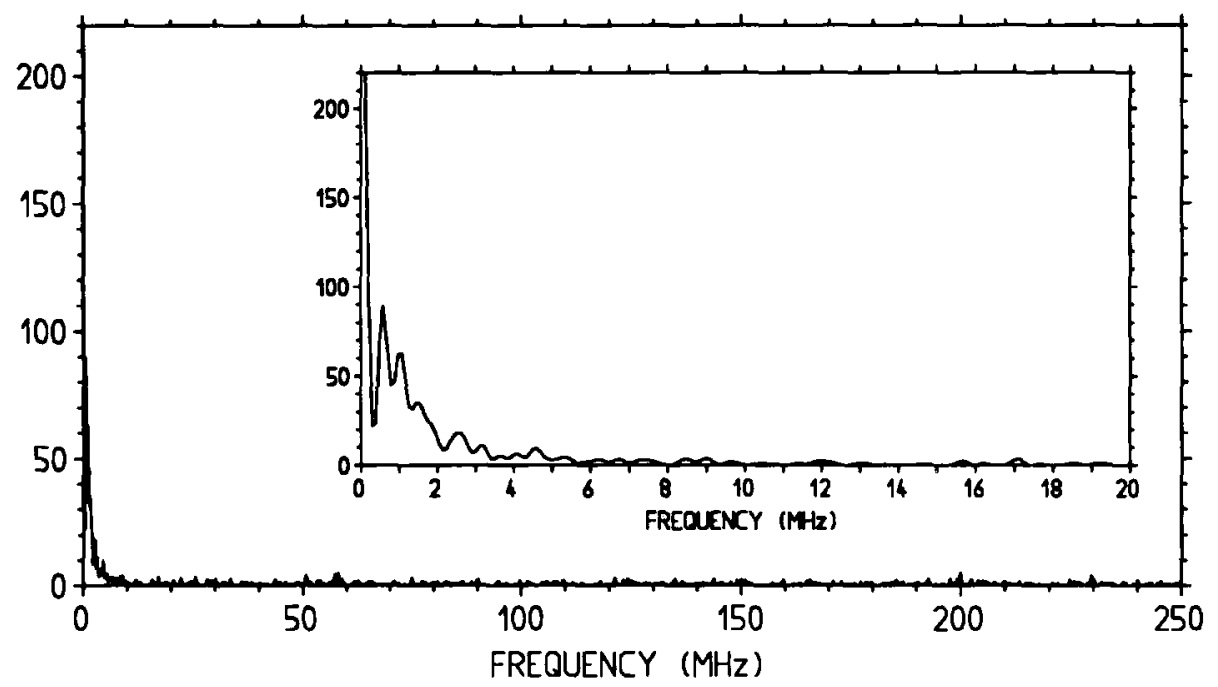

Fig. 4. Fourier power for negative muons in the $\mathrm{C}_{60}$ sample at $295 \mathrm{~K}$ and $0.8 \mathrm{mT}$. (Insert with the first $20 \mathrm{MHz}$ expanded.) No signal indicating the existence of a chemical species with an unpaired electron coupling to the $\mu^{-}$is visible.

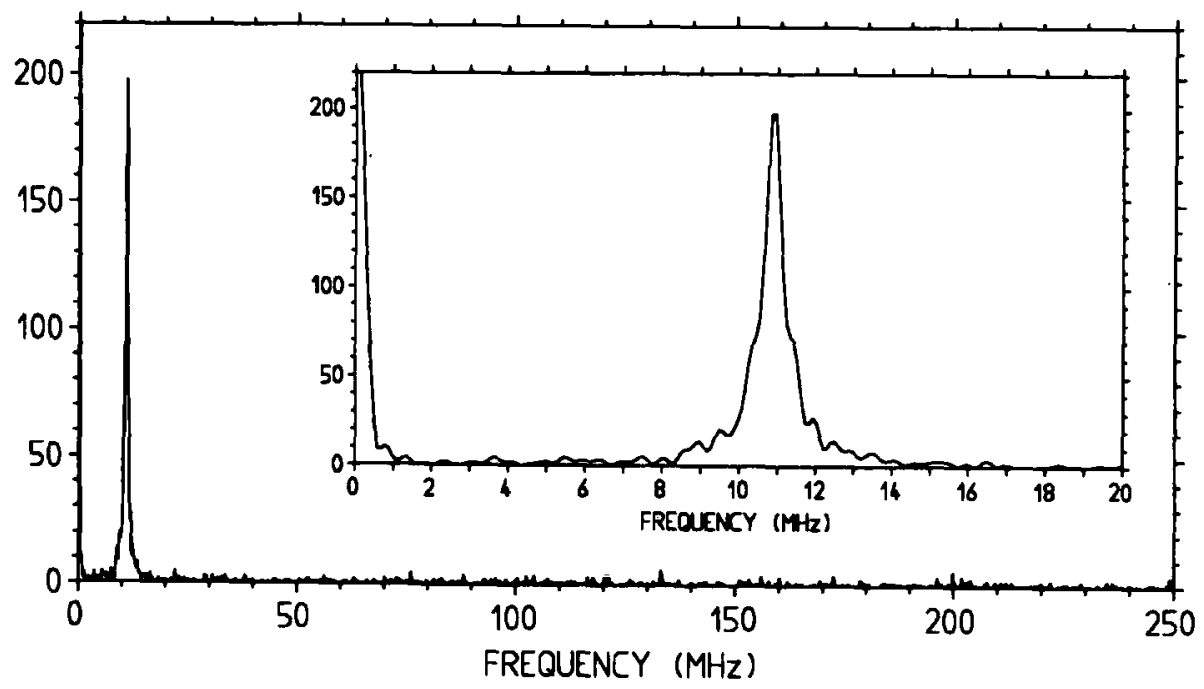

Fig. 5. Calibration in $\mathrm{C}_{60}$ at $0.8 \mathrm{mT}$. Fourier power for positive muons in the $\mathrm{C}_{60}$ sample at $295 \mathrm{~K}$ and $0.8 \mathrm{mT}$. The insert shows the first $20 \mathrm{MHz}$ expanded. The $10.9 \mathrm{MHz}$ signal (muonium + muonic radicals) is fitted with $A=4.4(2) \%$ and $\lambda=2.0(1) \mu \mathrm{s}^{-1}$. A $\mu^{+}$ run at $10 \mathrm{mT}$ indicates $A=3.3(1) \%$ for the $\mu^{+}$signal. 
In contrast Fig. 5 shows quite clearly the muonium and muonic radical low field signal obtained from $\mu^{+}$in the same sample, the combined asymmetry possessing a fitted value of $4.4(2) \%$. The result implies that any long lived $\mu^{-}$signal must possess an asymmetry well below $1 \%$. The more or less complete loss of $\mu^{-}$asymmetry is probably a result of rapid electron spin relaxation (of the order of the hyperfine splitting frequency) or of the modulation of the hyperfine interaction due to the tumbling of the $\mathrm{C}_{60-n}$ "B" molecule at room temperature.

Inquiring more deeply one can ask what happens to the sixth electron of the $\left(\mathrm{C} \mu^{-}\right) 1 \mathrm{~s}^{2} 2 \mathrm{~s}^{2} 2 \mathrm{p}^{2}$ atom which may be lost since the pseudo "boron" atom has only an effective "nuclear" charge of +5 . Since it is known that the electron affinity of the $\mathrm{C}_{60}$ molecule is quite strong, it is conceivable that this electron may remain attached to the $\mathrm{C}_{60-n}$ " $\mathrm{B}$ " molecule in an unpaired state and may thus interact strongly with the other unpaired electron on the "B"-site without actually forming a spin singlet configuration.

\section{References}

[1] R.L. Garwin, L.M. Ledermann and M. Weinrich, Phys. Rev. 106 (1957) 1415.

[2] A.O. Weissenberg, Muons (North Holland, Amsterdam, 1967).

[3] J. Major, private communication.

[4] D. Bakowies and W. Thiel, J. Am. Chem. Soc. 113 (1991) 3704. 